\title{
Correction to: Validity of transit time-based blood pressure measurements in patients with and without heart failure or pulmonary arterial hypertension across different breathing maneuvers
}

\author{
Sara Becker ${ }^{1}$. Jens Spiesshoefer ${ }^{1} \cdot$ Tobias Brix $^{2} \cdot$ Izabela Tuleta $^{3} \cdot$ Michael Mohr $^{4} \cdot$ Michele Emdin $^{5,6}$. \\ Matthias Boentert ${ }^{1}$. Alberto Giannoni ${ }^{5,6}$ \\ Published online: 6 June 2020 \\ (C) Springer Nature Switzerland AG 2020
}

Correction to: Sleep and Breathing (2020) 24:221-230 https://doi.org/10.1007/s11325-019-01848-w

Following the completion of the final manuscript version, Ms Sara Becker (who is the shared co-author of the article) prepared her doctoral thesis which is in part based on the published results. While we again went through the raw data we found minor errors which affect the online supplemental figures S1 and S2. These data relate to the capacity of PTT-based blood pressure (BP) recordings to detect changes in systolic and diastolic blood

The online version of the original article can be found at https://doi.org/ 10.1007/s11325-019-01848-w

Electronic supplementary material The online version of this article (https://doi.org/10.1007/s11325-020-02120-2) contains supplementary material, which is available to authorized users.

Jens Spiesshoefer

jens.spiesshoefer@ukmuenster.de

1 Respiratory Physiology Laboratory, Department of Neurology with Institute for Translational Neurology, University of Muenster, Muenster, Germany

2 Institute of Medical Informatics, University of Muenster, Muenster, Germany

3 Department of Cardiology I, University Hospital Muenster, Muenster, Germany

4 Department of Medicine A, Hematology, Oncology and Pulmonary Medicine, University Hospital Muenster, Muenster, Germany

5 Cardiovascular Medicine Division, Fondazione Toscana Gabriele Monasterio, National Research Council, CNR-Regione Toscana, Scuola Superiore San't Anna, Pisa, Italy

6 Institute of Life Sciences, Scuola Superiore Sant'Anna, Pisa, Italy pressure in different cohorts of patients. These errors occured when data were transformed from MS Excel to Sigma-Plot tables. Data and figures included in the main article are not affected. After correction of the supplemental data the respective figures were now revised. Importantly, these corrections also influence the discussion and conclusion sections of the main article. While it is still true that HF patients showed more artifacts with the PTT-based recordings, the statement that the only significant group difference regarding the two methods for detection DBP changes was found in HFrEF patients is incorrect. Thus, the following paragraphs of the results and discussion sections were also revised:

\section{Results section (last paragraph)}

Initial text:

Overall sensitivity to detect both increases and decreases in BP was similar for changes in SBP (bias $<1 \mathrm{mmHg}, \mathrm{SD} \approx 15 \mathrm{mmHg}$ across all three groups, Fig. S1) than for DBP (bias $<1 \mathrm{mmHg}$, $\mathrm{SD} \approx 14 \mathrm{mmHg}$, Fig. S2). Changes in DBP were detected with most variability in patients with $\mathrm{HFrEF}$ (bias $<1 \mathrm{mmHg} \mathrm{SD}$ $18.1 \mathrm{mmHg}$ vs. bias $<1 \mathrm{mmHg} 10.1 \mathrm{SD}$ and bias $<1 \mathrm{mmHg}$ SD $11.0 \mathrm{mmHg}$ in healthy volunteers and PAH patients, respectively).

Correction:

Overall sensitivity to detect both increases and decreases in $\mathrm{BP}$ was similar for changes in SBP (bias $<1.5 \mathrm{mmHg}, \mathrm{SD} \approx$ $11 \mathrm{mmHg}$ across all three groups, Fig. S1) and for changes in DBP (bias $<1.5 \mathrm{mmHg}, \mathrm{SD} \approx 8 \mathrm{mmHg}$, Fig. S2). In healthy volunteers, changes in both SBP and DBP were detected by PTT-based measurement with less variability than in patients with HF or PAH, but differences were not significant.

2. Discussion section: 
a. Page 8: The sentence "Plus, the only significant difference between groups in terms of bias between the two methods for the detection of changes in DBP was found in HFrEF patients too" has to be considered incorrect according to the revised data set.

Thus, respective paragraph should read as follows :

"Similar to previous reports $[8,9]$, we found that PTTderived BP measurement tended to be less effective and accurate in patients with HFrEF. We also found that the only subjects for whom calibration of PTT-based BP measurement against oscillometry was impossible $(n=3)$, all had HF and all were in atrial fibrillation. In addition, overall limits of agreement between the two methods were widest in HFrEF patients and for diastolic blood pressure values, in particular. Furthermore, PTT-based BP recordings appear to be less sensitive to changes in both $S B P$ and $D B P$ in $p a-$ tients with $H F$ and PAH when compared with healthy individuals. There are a number of factors that might account for the observation that in PTT-derived BP is less accurate in patients with HFrEF. First, Woolam and colleagues were the first to document the influence of cardiovascular disease on PWV [26]. Clearly, impaired arterial wall elasticity may impede accurate detection of the peripheral pulse wave. This would also explain underestimation of systolic BP in PAH patients. Secondly, impaired pump function (left sided in HFrEF patients or right sided in PAH patients) makes the pulse wave less pronounced and therefore harder to detect peripherally."

b. Page 9 (last paragraph): "In conclusion, PTT- based BP measurements are clinically acceptable, though slightly inaccurate over a period of $1 \mathrm{~h}$, including during SDBlike breathing patterns. This applies to healthy volunteers, and to patients with PAH or HFrEF patients. It should be noted that HFrEF patients often have arrhythmias and that this may lead to more difficulties regarding the calibration of PTT-based BP measurement against oscillometry. "

Publisher's note Springer Nature remains neutral with regard to jurisdictional claims in published maps and institutional affiliations. 